\title{
Relapsing Tumefactive Demyelination: A Case Report
}

\author{
Ibrahim Omerhodžić ${ }^{1}$, Almir Džurlić ${ }^{1}$, Dino Lisica ${ }^{1}$, Nevena Mahmutbegović ${ }^{2}$, \\ Maida Nikšićc ${ }^{3}$ Nurija Bilalovićt ${ }^{4}$ Enra Suljić ${ }^{2}$
}

${ }^{1}$ Neurosurgery Clinic, Clinical Center of Sarajevo University, Sarajevo, Bosnia and Herzegovina, ${ }^{2}$ Neurology Clinic, Clinical Center of Sarajevo University, Sarajevo, Bosnia and Herzegovina, ${ }^{3}$ Radiology Clinic, Clinical Center of Sarajevo University, Sarajevo, Bosnia and Herzegovina, ${ }^{4}$ Department of Pathology, Clinical Center of Sarajevo University, Sarajevo, Bosnia and Herzegovina

Correspondence: ibrahimomerhodzic74@gmail.com Tel: + 38761736486

Fax: + 38733297820

Received: 5 February 2018

Accepted: 27 June 2018

Key words: Multiple Sclerosis - Relapsing Remitting - Demyelinating.
Objective. We present a case of relapsing tumefactive demyelination in a young female patient, that posed a real diagnostic challenge, with a heterogeneous clinical picture, atypical for multiple sclerosis (MS) presentation, and neuroradiological manifestations with a high suspicion of neoplastic diseases. Case Report. An 18-year old female patient presented to our Neurosurgical Out-patients' Clinic with symptoms atypical for multiple sclerosis, unremarkable neurological deficit, one tumefactive lesion on MRI, followed by relapse and another two lesions within a period of six months. We decided to perform biopsy of the tumefactive lesion with compressive effect. Serological and clinical data were negative for MS, and the patient did not respond well to corticosteroid therapy. Fresh frozen tumor tissue aroused a strong suspicion of gemistocytic astrocytoma, so total resection was done, but the definitive pathohistological examination confirmed tumefactive demyelination. Conclusion. For clinicians, it is important to consider demyelinating disease in the differential diagnosis of a tumorlike lesion of the central nervous system, in order to avoid invasive and potentially harmful diagnostic procedures, especially in younger patients.

\section{Introduction}

Tumefactive demyelination is rare atypical inflammatory demyelinating syndrome, predominantly affecting young people, with an estimated prevalence of $1-3 / 1000$ cases and an annual incidence of 0.3/100,000 (1). Magnetic resonance imaging (MRI) usually specifies demyelinating brain lesions $2 \mathrm{~cm}$ or larger in size, often with perilesional edema, mass effect and incomplete ring enhancement, unlike the typical small, homogenous, well demarcated plaques of multiple sclerosis (2).

The clinical presentation may vary from a benign monophasic form to an acute fulmi- nant variant with lethal outcome, presenting with atypical multiple sclerosis symptoms such as: headache, cognitive abnormalities, mental confusion, aphasia, apraxia and/or seizures (3). Literature data most commonly report sensory deficit, motor weakness and cognitive impairment as the initial clinical presentation (4).

Tumefactive demyelination itself often represents a diagnostic challenge to neurologists and radiologists, with a misleading clinical picture, resulting in up to $10 \%$ incorrect diagnoses, mostly diagnosis of neoplastic diseases, infective diseases, other inflammatory disorders (5) or even a stroke (6). Therefore, histopathological confirma- 
tion is sometimes unavoidable for confirmation of the diagnosis.

We present a case of relapsing tumefactive demyelination in a young female patient, that posed a real diagnostic challenge, with a heterogeneous clinical picture, atypical for multiple sclerosis presentation, and neuroradiological manifestations with a high suspicion of neoplastic diseases.

\section{Case Report}

An 18-year old female Bosnian patient presented to our Neurosurgical Outpatients' Clinic in November 2014 with a 2 month
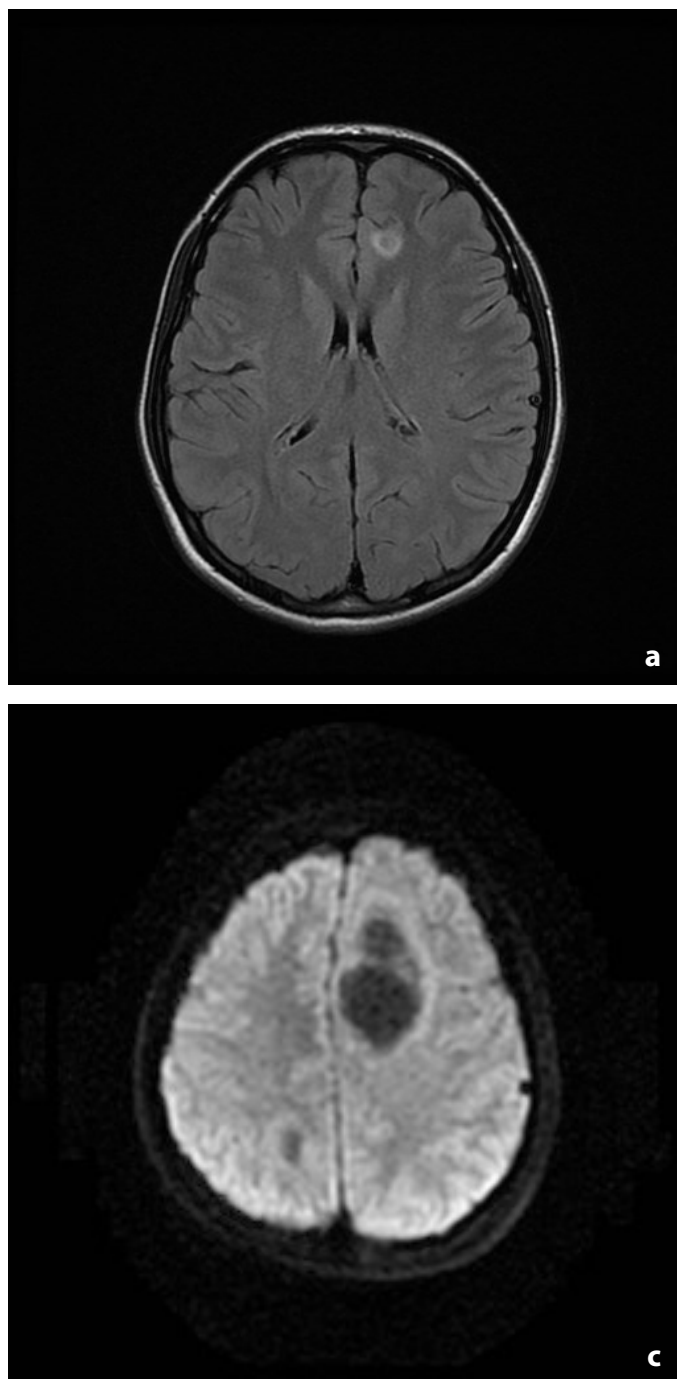

history of transient visual loss and headache. A head MRI (Figure 1a) showed a solitary lesion up to $11 \mathrm{~mm}$ in diameter in the right frontoparietal subcortical white matter, while the initial MRI scans of the brain and cervical spine, done two months earlier, were without pathological changes. At that time, she was admitted to the neurological ward, and she felt numbness followed by progressive weakness of her lower extremities. CSF examination showed elevated protein level (3.22 g/l, IgG index 63.7), EMNG findings showed severe generalized sensorimotor polyneuropathy of the axonal-demyelinating type, and she was diagnosed with acute

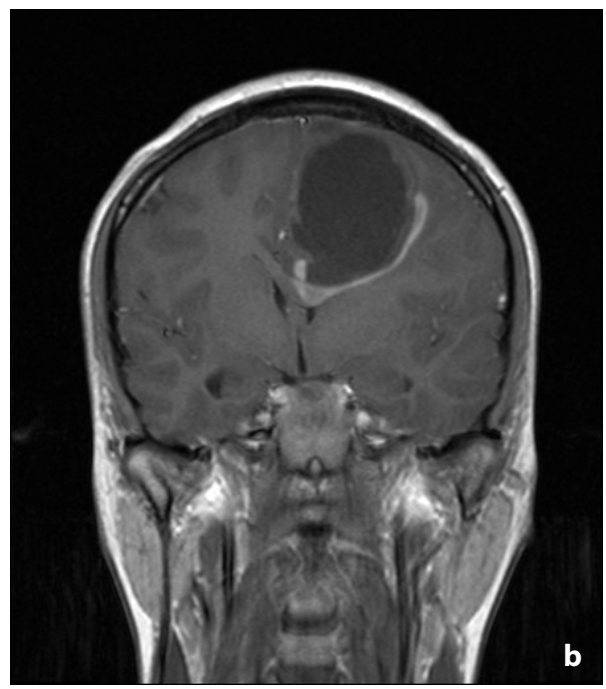

Figure 1. a) Brain MRI performed on $10^{\text {th }}$ November 2014, Axial T2 showing a solitary lesion without contrast enhancement. (Flair Axial). b) Preoperative brain MRI showing enlargement of the lesion to more than $7 \mathrm{~cm}$ in AP diameter and its compressive effect on surrounding structures. c) The emergence of new zones of changed signal intensity in the left frontoparietal region immediately next to the extensive zone of signal intensity changes, with perifocal edema in the left frontoparietal supraventricular region, and right parietal parasagital region. There is an impression of discrete reduction in the peripheral edema extension, but also of the same extension of the tumefactive lesion to the left (DWI).

polyradiculoneuritis and successfully treated with plasma exchange (5 cycles). 
A follow-up MRI, done six months later, revealed enlargement of the lesion previously described to $57 \mathrm{~mm} \times 32 \mathrm{~mm}$, with postcontrast ring enhancement (Figure 1b). MRI of the cervical spine was normal. Serological findings were all negative. The IgG index was high and oligoclonal bands were negative. HIV status was not checked. The patient was tested for aquaporin 4 antibodies and they were negative. She was treated with pulse corticosteroid therapy, but 2 weeks after, follow-up MRI (Figure 1c) showed enlargement of the lesion previously described, it was now $70 \mathrm{~mm}$ in AP diameter with compressive effect and medio-sagittal shift, and there were two new lesions, located in the left frontoparietal and right parietal white matter, sized $10 \mathrm{~mm}$ in diameter.

We decided to perform a surgical procedure to achieve decompression and to take tissue for pathohistological diagnosis. The neurosurgeon (IO) performed frontopari-
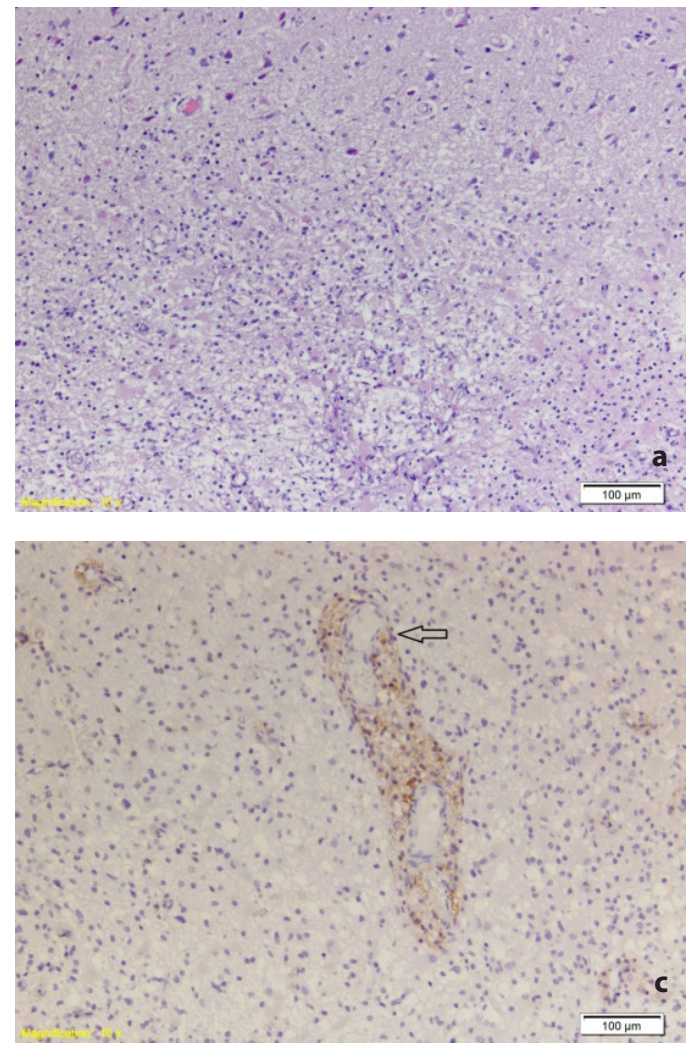

etal craniotomy. Intraoperatively, we found a tumor-like formation with solid edges, a central cyst with a white reticular wall, partially well limited from the brain tissue, partially infiltrating the surrounding brain tissue. Fresh frozen tumor tissue biopsy showed a strong suspicion of gemistocytic astrocytoma, and gross total resection of the lesion was done, but the definitive pathohistological examination confirmed the diagnosis of demyelination (Figure 2a-c).

In September 2015 the patient was admitted again to the Neurological Department due to neurological deterioration (EDSS 6). A new MRI showed the progression of the changes previously described and the emergence of new ones (Figure 3a). After pulse corticosteroid therapy was administrated, there was complete withdrawal of the neurological deficit. She started interferon therapy, and the follow-up MRI (October 2016) showed almost complete resolution of

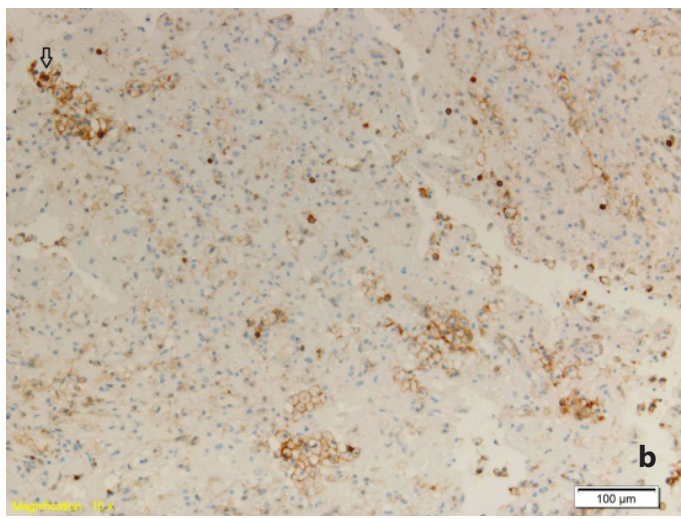

Figure 2. a) Hypercellular lesions with confluent demyelination, abundant foamy macrophages containing myelin debris, reactive astrogliosis, 'relative' axonal preservation and variable perivascular and parenchymal lymphocytic inflammation. JC virus chromogenic in situ hybridisation was negative. b) The results obtained from immunohistochemical analysis were used to determine demyelinating changes, central necrosis, macrophage infiltration, angiogenesis and perivascular lymphocytic cuffing (CD 4). c) Immunohistochemical staining for CD 163 (magnification $\times 10$ ). Perivascular cuffing inflammatory cells and macrophages contain myelin debris within their cytoplasm. 

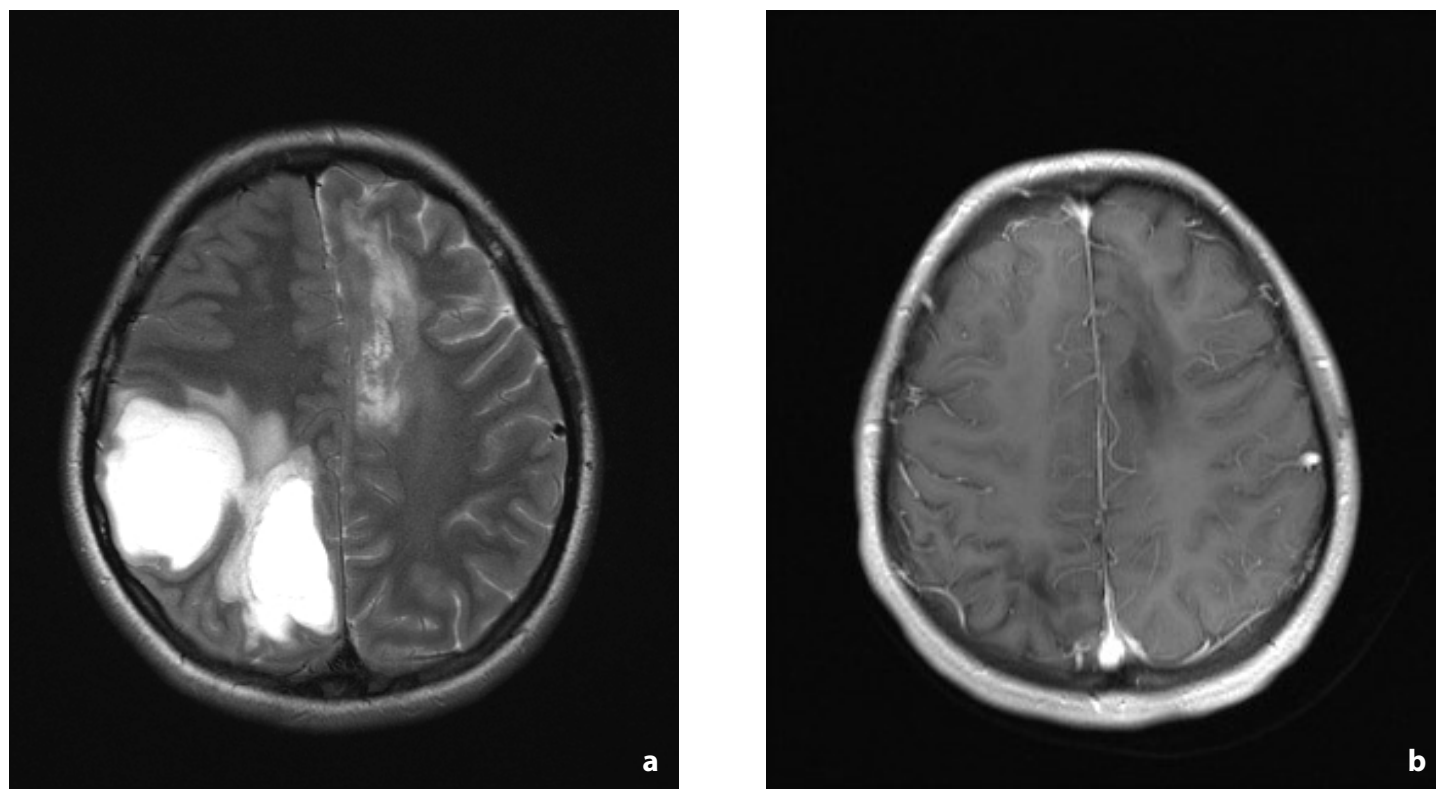

Figure 3. a) Brain MRI showing further evolution of the tumefactive lesions (T2 axial). b) Brain MRI showing resolution of multiple lesions (T2 axial).

the lesions previously described (Figure $3 b$ ). She has been stable since then and no further relapse has been reported.

\section{Discussion}

Tumefactive demyelination may occur as solitary or multiple intracranial masses larger than $2.0 \mathrm{~cm}$ in diameter, presenting as a distinct demyelinating disease or sustained in other demyelinating process, such as multiple sclerosis, acute disseminated encephalomyelitis, or neuromyelitis optica spectrum disorders (7). Gender predilection has not been well determined, although the incidence has been suggested to be higher in males (8). However, according to the literature review by Lucchinetti et al. (9), most of the reported patients were females with an average age of 37 , and the typical localization of tumefactive lesions was in the frontal or parietal lobes, as in our case. In terms of location, lesions occur most frequently in the frontal or parietal lobes, with variable effects on the gray matter, and often adjacent spread into the corpus callosum (10).
Tumefactive demyelination can pose real diagnostic difficulties in cases of a high suspicion of neoplastic disease. Large tumefactive lesions may be indistinguishable from neoplasms on MRI, as both can lead to mass effect/edema, a hypo-intense rim on T2 weighted scans, venular enhancement, and a variable degree of ring-enhancement (3). DWI and ADC sequences might be crucial for correct diagnosis, as the changes on diffusion-weighted imaging evolve rapidly, unlike stable tumorous or abscess lesions with no apparent diffusion coefficient restriction (7). Additionally, the use of FDG-PET scanning can be useful, as the degree of hypermetabolism of acute demyelinating lesions appears to be smaller compared to neoplastic lesions, but in the case of the unavailability of FDG-PET, a combination of non-contrast brain CT in addition to MRI can improve the diagnostic accuracy of tumefactive demyelination (7). The CSF may be normal or have high levels of immunoglobulin G (IgG) index and oligoclonal bands. (11) Abnormal visual (VEPs) and somatosensory evoked 
potentials may present in $33-60 \%$ of cases with tumefactive demyelination (9).

In a few cases biopsy is unavoidable, although frozen sections rarely suggest a diagnosis of a demyelinating disease. The target of biopsy should always be the wall of the tumefactive demyelinating lesion, unlike in tumors, where the central core of the lesion is more important (12). The histological features may mimic a tumor, including hypercellularity, astrocyte pleomorphism, variable nuclear atypia, a rare mitotic figure, and occasionally necrosis or cystic changes. The presence of Creuztfeldt-Peters cells may be mistaken for mitotic glial cells. However, the presence of large numbers of infiltrating macrophages in the setting of myelin loss and relative axonal preservation should confirm the diagnosis of an inflammatory demyelinating disease (13). With the development of neuroimaging, biopsy can be avoided; it is used mostly in patient without a pre-existing diagnosis of MS, with indecisive imaging, and negative oligoclonal bands, as it was in our case report.

Our patient was previously diagnosed with acute polyradiculoneuritis and successfully treated with plasma exchange, with complete withdrawal of neurological deficit. Although both acute polyradiculoneuritis and demyelinating diseases are of immunemediated origin, their co-existence is very rare, which makes the diagnostic dilemma even greater. Two studies have reported that the prevalence of Guillain-Barre syndrome (GBS) was higher in the MS population than in a control group $(14,15)$, however, only one study had statistically significant findings (15). The diagnosis of GBS, at our center, was based on the well-established diagnostic criteria and the subsequent development of tumefactive demyelination may suggest a kinship between these two entities.

Literature data regarding the course of tumefactive demyelination and prognoses are insufficient. Acute treatment includes intravenous methylprednisolone and/or plasma exchange, rituximab and natalizum$\mathrm{ab}$, followed by immunomodulatory agents (16). Some patients have a monophasic selflimited course, but recurrence of the disease with new tumefactive lesions is not unusual (17). According to Lucchineti et al. (9), the median time interval was 4.8 years until the second relapse. The neuroanatomical sites of recurrent demyelinating lesions in the present case were the contralateral frontoparietal and the right parietal white matter. Khan et al. (17) reported a case of a 34 year old male with contralateral recurrence of tumefactive lesions, while some authors suggest that new tumefactive lesions occur most commonly at the site of the index lesion (10).

Due to the limited data available in the literature, the prognostic significance of recurrent tumefactive lesions remains undetermined. Many patients demonstrate a monophasic self-limited course, while others experience relapse despite identical imaging features (10). It has not been established whether the neuroanatomical mechanism of the recurrence represents a repercussion of different underlying pathogenesis, and whether it impacts patient's prognosis (9). It has been reported that lesions larger than $5 \mathrm{~cm}$ were associated with greater disability at follow-up (9). On the other hand, Wattamwar and coauthors described the significant long-term functional recovery of 14 patients from their large demyelinating lesions (18). Some studies report clinical and radiological improvement of tumefactive demyelinating lesions, with no future development of typical recurrent relapsing MS (16). In contrast, a few authors report the development of clinically definitive MS (9).

\section{Conclusion}

For clinicians, it is important to consider demyelinating disease in the differential diagnosis of a tumor-like lesion of the central nervous system, in order to avoid invasive and potentially harmful diagnostic proce- 
dures, especially in younger patients with atypical clinical or imaging features.

\section{What is already known in this topic}

Tumefactive demyelination is a rare entity among the demyelinating group of diseases as a leading cause of progressive neurological deficit in young adults. Although there are no pathognomonic signs to indicate tumefactive demyelination, advances in neuroimaging are often sufficient in recognition of this peculiar manifestation of inflammatory demyelinating diseases.

\section{What this study adds}

This study emphasis that many cases of tumefactive demyelination can be monitored simply, clinically and radiologically. However, in the case of atypical clinical or imaging features, surgical biopsy should not be delayed, in order to avoid diagnostic speculation, and to implement appropriate therapy.

Authors' contributions: Conception and design: IO and DL; Acquisition, analysis and interpretation of data: ADŽ, NM, MN, NB and DL; Drafting the article: ADŽ, NM and DL; Revising it critically for important intellectual content: IO and ES; Approved final version of the manuscript: IO and ES.

Conflict of interest: The authors declare that they have no conflict of interest.

\section{References}

1. Frederick MC, Cameron MH. Tumefactive Demyelinating Lesions in Multiple Sclerosis and Associated Disorders. Curr Neurol Neurosci Rep. 2016;16(3):26.

2. Barkhof F, Rocca M, Francis G, Van Waesberghe $\mathrm{JH}$, Uitdehaag BM, Hommes OR, et al. Validation of diagnostic magnetic resonance imaging criteria for multiple sclerosis and response to interferon betala. Ann Neurol. 2003;53(6):718-24.

3. Dolic K, Bilic I, Buca A, Radovic D, Titlic M. Differentiation of Tumefactive Demyelinating Lesions from Metastatic Brain Disease with FDG PET-CT: A Case Report. J Mult Scler. 2014;1:108.

4. Kuan Y-C, Wang K-C, Yuan W-H, Tsai C-P. Tumefactive Multiple Sclerosis in Taiwan. PLoS ONE. 2013;8(7):e69919.

5. Jitawatanarat P, Tingpej B, Deringer P. Tumefactive Multiple sclerosis. BJMP. 2011;4(2):a419.

6. Yacoub HA, Al-Qudahl ZA, Lee HJ, Baisre A, Souayah N. Tumefactive Multiple Sclerosis presenting as Acute Ischemic Stroke. J Vasc Interv Neurol. 2011;4(2):21-3.

7. Hardy TA, Reddel SW, Barnett MH, Palace J, Lucchinetti CF, Weinshenker BG. Atypical inflamma- tory demyelinating syndromes of the CNS. Lancet Neurol. 2016;15(9):967-81.

8. Kobayashi M, Shimizu Y, Shibata N, Uchiyama S. Gadolinium enhancement patterns of tumefactive demyelinating lesions: correlations with brain biopsy findings and pathophysiology. J Neurol. 2014;261(10):1902-10.

9. Lucchinetti CF, Gavrilova RH, Metz I, Parisi JE, Scheithauer BW, Weigand S, et al. Clinical and radiographic spectrum of pathologically confirmed tumefactive multiple sclerosis. Brain. 2008;131(Pt 7):1759-75.

10. Nagappa M, Taly AB, Sinha S, Bharath RD, Mahadevan A, Bindu PS, et al. Tumefactive demyelination: clinical, imaging and follow-up observations in thirty-nine patients. Acta Neurol Scand. 2013;128(1):39-47.

11. Seewann A, Enzinger C, Filippi M, Barkhof F, Rovira A, Gass A, et al. MRI characteristics of atypical idiopathic inflammatory demyelinating lesions of the brain : A review of reported findings. J Neurol. 2008;255(1):1-10.

12. Neelima R, Krishnakumar K, Nair MD, Kesavadas C, Hingwala DR, Radhakrishnan VV, et. Tumefactive demyelinating lesions: a clinicopathological correlative study. Indian J Pathol Microbiol. 2012;55(4):496-500.

13. Kuhlmann T, Lassmann H, Brück W. Diagnosis of inflammatory demyelination in biopsy specimens: a practical approach. Acta Neuropathol. 2008;115(3):275-87.

14. Edwards LJ, Constantinescu CS. A prospective study of conditions associated with multiple sclerosis in a cohort of 658 consecutive outpatients attending a multiple sclerosis clinic. Mult Scler. 2004;10(5):575-81.

15. Langer-Gould A, Albers KB, Van Den Eeden SK, Nelson LM. Autoimmune diseases prior to the diagnosis of multiple sclerosis: a population-based case-control study. Mult Scler. 2010;16(7):855-61.

16. Hardy TA, Chataway J. Tumefactive demyelination: an approach to diagnosis and management. J Neurol Neurosurg Psychiatry. 2013;84(9):1047-53.

17. Khan MN, Guranda M, Essig M. Contralateral recurrence of tumefactive demyelination. Neuroradiol J. 2015;28(5):493-7.

18. Wattamwar PR, Baheti NN, Kesavadas C, Nair M, Radhakrishnan A. Evolution and long term outcome in patients presenting with large demyelinating lesions as their first clinical event. J Neurol Sci. 2010;297(1-2):29-35. 